\title{
Research Article \\ Risk Factors for Cognitive Impairment in Patients with Type 2 Diabetes
}

\author{
Lin Sun, ${ }^{1}$ Xue Diao, ${ }^{2}$ Xiaokun Gang $\mathbb{D}^{1},{ }^{1}$ You $\mathrm{Lv},{ }^{1}$ Xue Zhao, ${ }^{1}$ Shuo Yang, ${ }^{1}$ Ying Gao, \\ and Guixia Wang (iD \\ ${ }^{1}$ Department of Endocrinology and Metabolism, The First Hospital of Jilin University, Changchun, 130021 Jilin Province, China \\ ${ }^{2}$ Department of Endocrinology and Metabolism, The University of Hong Kong-Shenzhen Hospital, Shenzhen 518000, \\ Guangdong Province, China
}

Correspondence should be addressed to Guixia Wang; gwang168@jlu.edu.cn

Received 6 January 2020; Accepted 7 April 2020; Published 24 April 2020

Academic Editor: Dario Pitocco

Copyright () 2020 Lin Sun et al. This is an open access article distributed under the Creative Commons Attribution License, which permits unrestricted use, distribution, and reproduction in any medium, provided the original work is properly cited.

\begin{abstract}
Objectives. To investigate the risk factors for cognitive impairment in Chinese type 2 diabetes mellitus (T2DM) patients of advanced age and to identify effective biomarkers of mild cognitive impairment (MCI) in these patients. Methods. Chinese T2DM patients ( $n=120)$ aged 50-70 years were divided into groups with impaired (mild, moderate, and severe) and normal cognitive function based on Montreal Cognitive Assessment and Mini-Mental State Examination scores. Data regarding demographic characteristics, clinical features of diabetes, biochemical markers, and metabolomics were collected. Results. Age, educational level, duration of diabetes, fasting blood glucose (FBG), HbA1c, total cholesterol (TC), triglyceride (TG), and 24-hour urine protein were significantly associated with cognitive impairment in T2DM patients of advanced age. The severity of fundus retinopathy and the incidence of macrovascular disease also differed significantly among the groups $(P<0.05)$. Metabolomics analysis suggested that increased levels of glutamate (Glu), phenylalanine (Phe), tyrosine (Tyr), proline (Pro), and homocysteine (Hcy) and a decreased level of glutamine (Gln) were significantly associated with cognitive impairment in the T2DM patients $(P<0.05)$. Receiver operating characteristic curve analysis demonstrated that Glu, Gln, Phe, and Pro levels were significant predictors of cognitive impairment in the T2DM patients. Conclusions. Age, educational level, duration of diabetes, and the levels of FBG, HbA1c, TC, TG, and 24-hour urine protein were considered as independent risk factors for cognitive impairment in older T2DM patients. Macrovascular and microvascular diseases also were closely associated with cognitive impairment in these patients. Together, Glu and Gln levels may represent a good predictive biomarker for the early diagnosis of cognitive impairment in T2DM patients.
\end{abstract}

\section{Introduction}

Type 2 diabetes mellitus (T2DM) is characterized by relative insulin deficiency and insulin resistance, and obesity and sedentary lifestyle are generally considered to be the major risk factors [1]. According to changes in socioeconomic factors and increased practice of unhealthy lifestyle habits, the prevalence of diabetes is increasing in developing and developed countries [2]. T2DM is associated with cognitive decline, and patients with diabetes the patients exhibit worse cognitive ability and more abnormalities on brain imaging than individuals without diabetes $[3,4]$. The prevalence of mild cognitive impairment (MCI) is particularly higher in T2DM patients older than 65 years [5]. Multiple long-term epidemiological studies have implicated T2DM as a risk factor for cognitive dysfunction and dementia in the elderly $[6,7]$.

The mechanisms of cognitive function decline and brain structural abnormalities in T2DM patients remain incompletely understood. However, research has identified particular risk factors that promote the occurrence of MCI in T2DM patients, including vascular risk factors, macrovascular diseases, microvascular complications, poor glycemic control, hyperinsulinemia, increased oxidative stress, accumulation of amyloid-beta peptide and tau hyperphosphorylation, and nerve growth factor deficiency $[3,7,8]$. Currently, there are no specific measures for preventing or treating cognitive 
deficits in diabetic patients, and the importance of such impairment often receives less attention than other complications of T2DM [9]. Given that interventions for cognitive impairment are reasonably effective when applied during the early stages [10], it is important to clarify the characteristics of MCI in T2DM patients and to identify the effective diagnostic markers of MCI in these patients.

In this cross-sectional study, we used the Montreal Cognitive Assessment (MoCA) and Mini-Mental State Exam (MMSE) to assess the cognitive function of T2DM patients aged 50-70 years. We aimed at determining the characteristics of cognitive impairment in T2DM patients in this age range as well as identifying potential risk factors and biomarkers from among the demographic and clinical characteristics of the patients included in this study. This information can support strategies for the early diagnosis of MCI in T2DM patients.

\section{Materials and Methods}

2.1. Patients and Study Design. The present study included 120 patients who were admitted to the Department of Endocrinology of the 1st Hospital of Jilin University between October 2017 and September 2018, according to in-hospital records stored in electronic databases.

The inclusion criteria for subjects were as follows: (1) age 50-70 years and (2) diagnosis of T2DM at least 3 years prior to enrollment according to the criteria of the Type 2 Diabetes Mellitus Prevention Guideline in China. The exclusion criteria were as follows: (1) acute cerebral illness within the previous 3 months; (2) significant sequel of previous cerebrovascular disease; (3) psychosis, Parkinson's disease, brain tumor, encephalitis, or epilepsy; (4) thyroid dysfunction, CO poisoning, syphilis, or other systemic diseases that could cause cognitive impairment; (5) alcohol dependence or drug abuse; (6) obvious anxiety and depression; and (7) a history of severe infection or acute diabetic complications.

2.2. Collection of Clinical Data. For all of the patients, we collected data for demographic and clinical characteristics, including age, gender, education level, body mass index (BMI), duration of T2DM, and history of smoking, alcohol drinking, and hypertension. Systolic blood pressure (SBP), diastolic blood pressure (DBP), and levels of fasting blood glucose (FBG), fasting C-peptide (FCP), urea nitrogen, glycosylated hemoglobin (HbAlc), total cholesterol (TC), triglyceride (TG), low-density cholesterol (LDL-C), and highdensity cholesterol (HDL-C) were measured. Data related to diabetic complications such as optic fundi (I-VI phase), 24-hour urine protein, and carotid ultrasonography (collected by the Color Doppler Department) also were collected. Blood samples were obtained after fasting and analyzed by the Department of Clinical Laboratory of the First Hospital of Jilin University. The HbA1c levels were determined using an Automatic Glycohemoglobin Analyzer, and the FBG, creatinine (CRE), blood urea nitrogen (BUN), LDL-C, HDL-C, TG, and TC levels were measured by an Automatic Biochemistry Analyzer Hitachi 7060C.
BMI was calculated as body weight $(\mathrm{kg})$ divided by the square of height $\left(\mathrm{m}^{2}\right)$.

2.3. Cognitive Testing. The MoCA scale and MMSE scale were applied to assess the neuropsychological situation of all participants. We selected the MoCA Chinese version, which is a one-page, 10 -minute, 30-point screening test to identify individuals with MCI. It includes the testing of visual space and executive functions, naming, memory, attention, language, abstract thinking, calculation, and orientation. A MoCA score $\geq 26$ indicates normal cognition.

The Chinese version of the MMSE is a 30-point questionnaire used clinically to measure cognitive impairment. Any score $<27$ indicates decreased cognitive function. The raw score should be corrected for educational attainment as follows: illiteracy $\leq 17$ points, primary school level $\leq 20$ points, secondary school level (including technical secondary school) $\leq 22$ points, and university degree (including junior college) $\leq 23$ points.

The 120 included patients were divided into four groups based on the MoCA and MMSE results: a normal cognitive function (NCF) group $(n=40, \mathrm{MoCA} \geq 26$, and MMSE $\geq$ 27), a mild cognitive impairment (MCI) group $(n=37$, MoCA $<26$, and $21 \leq \mathrm{MMSE} \leq 26$ ), a moderate cognitive impairment $(\mathrm{MoCI})$ group $(n=31, \mathrm{MoCA}<26$, and $10 \leq$ MMSE $\leq 20$ ), and a severe cognitive impairment (SCI) group $(n=12, \operatorname{MoCA}<26$, and $0 \leq \mathrm{MMSE} \leq 9)$.

2.4. Metabolomics Analysis. All patients fasted for 8 hours before the collection of venous blood samples in the early morning of the next day.

The following chemical reagents were used: acetonitrile (HPLC grade, Thermo Fisher), pure water (Thermo Fisher), 1-butanol (Sigma-Aldrich), acetyl chloride (Sigma-Aldrich), and NSK-A and NSK-B isotope internal standards (Cambridge). All standards were mixed and dissolved in $2 \mathrm{~mL}$ pure methanol for storage at $4^{\circ} \mathrm{C}$. A working solution was obtained by $100 \times$ dilution and used to extract metabolites. Amino acid and carnitine QC standards were purchased from Chromsystems.

For sample processing, a $3 \mathrm{~mm}$ diameter circle was punched from each dry paper blood spot and placed in a well of a Millipore multilayer 96-well plate (Millipore Corp, Billerica, MA, USA) for metabolite extraction. Then, $100 \mu \mathrm{L}$ of the working solution was added to each well. The 96-well plate was centrifuged at $1500 \mathrm{~g}$ for 2 minutes after gentle shaking for 20 minutes, and the filtrates were collected from the lower layer of the wells. Four blank wells are randomly selected in each plate, and in them, two low controls and two high controls were included individually. The quality control samples and filtrate samples were blown dry in pure nitrogen at $50^{\circ} \mathrm{C}$. The dry samples were derivatized in a mixture of $60 \mu \mathrm{L}$ acetyl chloride and 1-butanol (1:9 by volume) at $65^{\circ} \mathrm{C}$ for 20 minutes. The derived samples were dried, and each dried sample was reconstituted in $100 \mu \mathrm{L}$ of fresh mobile phase solution for metabolite analysis.

For the detection of metabolomics markers, direct injection mass spectrometric analysis was performed using the AB Sciex 4000 QTrap system (AB Sciex, Framingham, MA, 
USA). The ion source of the instrument is an electrospray ion source that scans all analytes in positive ion mode. The injection volume was $20 \mu \mathrm{L}$, the mobile phase was $80 \%$ aqueous acetonitrile, and the initial flow rate was $0.2 \mathrm{~mL} / \mathrm{min}$. Then, the flow rate was reduced to $0.1 \mathrm{~mL} / \mathrm{min}$ in $0.08 \mathrm{~min}$, kept constant for $1.5 \mathrm{~min}$, returned to $0.2 \mathrm{~mL} / \mathrm{min}$ in $0.01 \mathrm{~min}$, and then maintained for $0.5 \mathrm{~min}$. The ion spray voltage was $4.5 \mathrm{kV}$, the curtain air pressure was set to $20 \mathrm{psi}$, the ion source gas 1 and gas 2 were $35 \mathrm{psi}$, and the auxiliary heating gas temperature was $350^{\circ} \mathrm{C}$. The system was controlled, and data were collected by the Analyst v1.6.0 software (AB Sciex, Framingham, MA, USA). Data were processed using ChemoView 2.0.2 (AB Sciex, Framingham, MA, USA).

2.5. Statistical Analysis. Statistical analysis was performed using SPSS 18.0 software. Mean \pm standard deviation values were used to describe quantitative data if normally distributed, and the median (quartile), i.e., M (P25-P75), were used to describe the quantitative data if not normally distributed. For quantitative data with a normal distribution and homogeneity of variance, differences between groups were analyzed by one-way analysis of variance (ANOVA). To compare data with a nonnormal distribution or with homogeneity of variance, a nonparametric test was used. Multivariate logistic regression models were used to analyze risk factors associated with cognitive impairment in patients with T2DM. $P<0.05$ denoted statistical significance.

\section{Results}

3.1. Demographic and Clinical Characteristics. The demographic and clinical data of the patients are presented in Table 1. The 120 enrolled patients included 82 males $(68.3 \%)$ and 38 females (31.7\%). The average age of the patients was $64.43 \pm 15.29$ years. Seventy-five patients $(62.5 \%)$ had a history of hypertension, and 64 patients (53.3\%) had a history of smoking.

Among the four groups with differing levels of cognitive function, statistically significant differences were found in age, duration of diabetes, BMI, education level, occurrence of cardiovascular events, smoking, FBG, HbA1c, TC, TG, and 24-hour urine protein (all $P<0.05$ ).

3.2. Binary Logistic Regression Analysis of Baseline Data and MoCA Score. Binary logistic regression analysis was performed with age, duration of diabetes, SBP, DBP, BMI, educational level, occurrence of cardiovascular events, smoking, FBG, HbA1c, TC, TG, CRE, BUN, LDL-C, HDL-C, Homeostatic Model of Insulin Resistance (HOMA-IR), and 24-hour urine protein as independent variables and the incidence of cognitive impairment as the dependent variable. From this analysis, age, duration of diabetes, BMI, education, cardiovascular events, smoking, FBG, HbA1c, TC, TG, and 24hour urine protein differed significantly between T2DM patients with cognitive impairment and those with normal cognitive function (Table $2, P<0.05$ ).

3.3. Correlation between Baseline Characteristics and Cognitive Impairment in T2DM Patients. From the results of the binary logistic regression analysis, for the ordinal mul- tivariate logistic regression analysis, we set age, diabetes duration, BMI, education level, occurrence of cardiovascular events, smoking, FBG, HbA1c, TC, TG, and 24-hour urine protein as independent variables and the incidence of cognitive impairment as the dependent variable. The results showed that the following factors were independently associated with cognitive impairment in T2DM patients: age, educational level, duration of diabetes, FBG, HbA1c, TC, TG, and 24-hour urine protein (Table 3).

3.3.1. Correlation between Fundus Retinopathy and Cognitive Impairment in T2DM. Significant differences in the degree of fundus retinopathy were observed between the four groups $(P<0.05$; Table 4$)$.

3.3.2. Correlation between Macrovascular Diseases and Cognitive Impairment in T2DM. Significant differences in the severity of macrovascular diseases were observed between the four groups of T2DM patients $(P<0.05$; Table 5).

3.4. Correlation between Metabolomics Indicators and Cognitive Impairment in T2DM. The results for metabolomics indicators in all patients showed that glutamate (Glu), glutamine (Gln), phenylalanine (Phe), tyrosine (Tyr), proline (Pro), and homocysteine (Hcy) levels were statistically different between the four groups $(P<0.05)$. Of those, Glu, Phe, Tyr, Pro, and Hcy levels increased with the development of cognitive impairments, whereas the Gln level decreased (Table 6). The results of receiver operating characteristic (ROC) curve analysis (Figure 1) of the six amino acids suggested that Glu, Gln, Phe, and Pro levels may be meaningful in the diagnosis of cognitive dysfunction in patients with T2DM (Table 7). Combined analysis of Glu and Gln levels by ROC curve analysis suggested that a diagnosis made based on Glu and Gln is more meaningful for the early diagnosis of cognitive impairments in T2DM patients. Normal ranges for clinical and metabolomics indicators are shown in Table A1 in appendix materials. Correlation between the level of acylcarnitine and cognitive impairment in T2DM patients of advanced age was shown in Table A2.

\section{Discussion}

Greater cognitive decline occurs among older people ( $>50$ years of age) with T2DM than among the general nondiabetic population of the same age. The results of our present study show that the severity of cognitive impairment in these T2DM patients was closely related to age and education level. As T2DM patients age, brain atrophy and lacunar infarction are observed with greater frequency. In addition, T2DM patients may exhibit small and punctate white-matter lesions, decreased brain volume, altered vascular function, oxidative stress, and accumulation of glycation end products. Biessels et al. [3] summarized the relationship between cognitive decline and age in T2DM patients and determined that cognitive impairment in T2DM patients occurs mostly from the ages of 40-80 years, and particularly from $60-80$ years. The available evidence suggests a negative correlation between education and the incidence of dementia [5]. A potential explanation is that individuals with a higher 
TABle 1: Demographic and clinical characteristics of the 120 T2DM patients grouped according to the degree of cognitive impairment.

\begin{tabular}{|c|c|c|c|c|c|c|}
\hline Characteristics & NCF group $(n=40)$ & MCI group $(n=37)$ & MoCI group $(n=31)$ & SCI group $(n=12)$ & $F / \chi^{2}$ & $P$ value \\
\hline Age (y) & $53.01 \pm 2.43$ & $56.45 \pm 4.62$ & $59.54 \pm 3.16$ & $64.45 \pm 5.24$ & 11.243 & $0.012^{*}$ \\
\hline Duration of diabetes (y) & $5.12 \pm 1.13$ & $6.34 \pm 2.53$ & $8.13 \pm 2.16$ & $10.14 \pm 8.24$ & 14.35 & $0.008^{*}$ \\
\hline Smoking $n(\%)$ & $15(37.50 \%)$ & $18(48.65 \%)$ & $23(74.19 \%)$ & $9(75.00 \%)$ & 8.532 & $0.018^{*}$ \\
\hline Education level (y) & $33(82.50 \%)$ & $24(64.86 \%)$ & $12(38.71 \%)$ & $4(33.33 \%)$ & 9.135 & $0.013^{*}$ \\
\hline Cardiovascular event & $13(32.50 \%)$ & $15(40.54 \%)$ & $16(51.61 \%)$ & $7(58.33 \%)$ & 7.357 & $0.042^{*}$ \\
\hline TC (mmol/L) & $3.42(3.34,4.32)$ & $3.33(3.24,4.56)$ & $3.84(3.12,4.78)$ & $4.51(3.56,5.78)$ & 12.505 & $0.002^{*}$ \\
\hline TG (mmol/L) & $2.51(1.23,3.21)$ & $2.71(1.34,3.67)$ & $3.13(1.56,4.56)$ & $4.24(1.67,5.32)$ & 13.87 & $0.004^{*}$ \\
\hline HDL-C (mmol/L) & $0.83 \pm 0.58$ & $0.85 \pm 0.78$ & $0.94 \pm 0.67$ & $1.14 \pm 0.78$ & 2.201 & 0.117 \\
\hline LDL-C (mmol/L) & $2.13 \pm 0.86$ & $2.43 \pm 0.97$ & $2.84 \pm 0.87$ & $3.32 \pm 1.12$ & 2.35 & 0.102 \\
\hline BMI $\left(\mathrm{kg} / \mathrm{m}^{2}\right)$ & $23.2 \pm 1.5$ & $24.1 \pm 1.8$ & $25.2 \pm 2.1$ & $26.7 \pm 2.4$ & 1.824 & $0.048^{*}$ \\
\hline SBP (mmHg) & $124 \pm 21$ & $137 \pm 23$ & $135 \pm 19$ & $142 \pm 21$ & 3.955 & 0.138 \\
\hline DBP (mmHg) & $78 \pm 9$ & $82 \pm 10$ & $79 \pm 8$ & $84 \pm 11$ & 2.538 & 0.281 \\
\hline FBG $(\mathrm{mmol} / \mathrm{L})$ & $6.84 \pm 1.75$ & $6.93 \pm 2.25$ & $7.43 \pm 2.16$ & $8.83 \pm 2.36$ & 10.266 & $0.044^{*}$ \\
\hline $\mathrm{HbAlc}(\mathrm{mmol} / \mathrm{L})$ & $6.7 \pm 1.2$ & $6.9 \pm 1.4$ & $7.3 \pm 1.8$ & $7.7 \pm 1.9$ & 12.551 & $0.002^{*}$ \\
\hline AST (U/L) & $31.55(13.45,38.54)$ & $23.00(18.65,41.42)$ & $25.70(20.12,43.56)$ & & & \\
\hline $\operatorname{ALT}(\mathrm{U} / \mathrm{L})$ & $21.2(10.34,25.67)$ & $43.70(32.45,48.64)$ & 2.239 & 0.113 & 2.926 & 0.134 \\
\hline ALP (U/L) & $72.75(50.46,89.44)$ & $71.45(43.45,89.34)$ & $81.34(68.34,99.34)$ & $85.34(72.39,91.77)$ & 2.509 & 0.285 \\
\hline Prealbumin (g/L) & $0.26(0.21,0.43)$ & $0.27(0.22,0.34)$ & $0.21(0.15,0.29)$ & $0.25(0.17,0.23)$ & 4.982 & 0.544 \\
\hline Apo-A1 (g/L) & $0.93(0.67,1.31)$ & $0.95(0.71,1.23)$ & $1.05(0.73,1.22)$ & $1.03(0.75,1.32)$ & 3.456 & 0.436 \\
\hline Apo-B (g/L) & $0.96(0.94,1.03)$ & $0.98(0.95,1.04)$ & $0.83(0.78,0.88)$ & $0.92(0.85,0.97)$ & 2.456 & 0.754 \\
\hline $\mathrm{T} \operatorname{Bil}(\mu \mathrm{mol} / \mathrm{L})$ & $13.06 \pm 6.12$ & $11.45 \pm 5.38$ & $14.42 \pm 7.18$ & $13.45 \pm 6.10$ & 1.345 & 0.435 \\
\hline I Bil $(\mu \mathrm{mol} / \mathrm{L})$ & $10.41 \pm 3.24$ & $7.63 \pm 2.42$ & $8.91 \pm 3.24$ & $6.93 \pm 3.21$ & 2.452 & 0.654 \\
\hline D Bil $(\mu \mathrm{mol} / \mathrm{L})$ & $3.42(3.21,3.56)$ & $2.91(2.34,3.56)$ & $3.31(2.67,3.97)$ & $2.54(2.14,3.08)$ & 5.356 & 0.267 \\
\hline $\mathrm{TBA}(\mu \mathrm{mol} / \mathrm{L})$ & $3.41 \pm 1.34$ & $4.42 \pm 2.05$ & $3.56 \pm 1.42$ & $3.13 \pm 1.54$ & 1.375 & 0.135 \\
\hline WBC & $6.31(4.87,7.46)$ & $7.54(5.36,8.46)$ & $6.04(5.03,7.98)$ & $7.04(5.35,9.75)$ & 3.467 & 0.079 \\
\hline NE\% & $0.60(0.45,0.71)$ & $0.51(0.43,0.76)$ & $0.56(0.51,0.68)$ & $0.62(0.55,0.73)$ & 2.349 & 0.275 \\
\hline LY\% & $0.33(0.25,0.38)$ & $0.31(0.27,0.39)$ & $0.37(0.33,0.41)$ & $0.41(0.36,0.49)$ & 0.923 & 0.319 \\
\hline 24 h UP (g/24h) & $0.21 \pm 0.07$ & $0.26 \pm 0.05$ & $0.24 \pm 0.02$ & $0.41 \pm 0.05$ & 15.563 & $0.014^{*}$ \\
\hline $24 \mathrm{~h}$ mAlb (mg/24h) & $43.82 \pm 30.34$ & $56.36 \pm 40.23$ & $55.23 \pm 43.54$ & $65.34 \pm 46.32$ & 3.561 & 0.145 \\
\hline CRP (mg/L) & $3.35(2.34,5.42)$ & $4.36(3.45,5.78)$ & $3.22(2.45,4.67)$ & $3.52(2.67,4.66)$ & 2.345 & 0.542 \\
\hline $\mathrm{SCr}(\mu \mathrm{mol} / \mathrm{L})$ & $72.6(63.46,77.54)$ & $77.56(72.45,79.54)$ & $63.42(54.67,69.13)$ & $73.34(65.88,78.64)$ & 1.386 & 0.638 \\
\hline BUN (mmol/L) & $5.23(4.24,6.75)$ & $6.21(5.78,6.94)$ & $5.61(4.35,7.68)$ & $5.11(4.24,6.97)$ & 2.457 & 0.846 \\
\hline $\mathrm{UA}(\mu \mathrm{mol} / \mathrm{L})$ & $245.32 \pm 53.45$ & $342.54 \pm 43.53$ & $354.65 \pm 34.67$ & $367.34 \pm 64.45$ & 8.642 & 0.063 \\
\hline $\operatorname{Alb}(g / L)$ & $38.21(33.56,41.34)$ & $40.41(35.78,43.67)$ & $39.52(32.56,42.87)$ & $35.64(31.56,40.23)$ & 5.682 & 0.105 \\
\hline Glb (g/L) & $28.23(25.78,30.14)$ & $28.23(23.56,32.67)$ & $23.54(21.38,27.58)$ & $25.45(22.46,26.95)$ & 2.843 & 0.638 \\
\hline $\mathrm{CO}_{2} \mathrm{CP}(\mathrm{mmol} / \mathrm{L})$ & $25.62(23.55,27.54)$ & $25.33(21.45,29.45)$ & $26.29(24.56,29.76)$ & $27.23(25.32,30.12)$ & 0.468 & 0.462 \\
\hline $\mathrm{CHE}(\mathrm{U} / \mathrm{L})$ & $9456.37 \pm 2539.60$ & $9228.10 \pm 2134.96$ & $9153.15 \pm 2671.12$ & $9423.19 \pm 2751.13$ & 0.246 & 0.723 \\
\hline
\end{tabular}

${ }^{*} P<0.05$. Abbreviations: TC: total cholesterol; TG: triglyceride; HDL-C: high-density lipoprotein cholesterol; LDL-C: low-density cholesterol.

education level and knowledge workers have a higher synaptic density in the cortex, which increases the brain's storage capacity, and thus, the symptoms of dementia are delayed for 4-5 years.

As the duration of T2DM increases, macrovascular and microvascular diseases, oxidative stress damage, and insulin resistance are likely to increase the damage to neurons. Logroscino et al. [11] found that cognitive decline in T2DM patients was positively correlated with the duration of diabetes, and the risk of cognitive impairment was significantly increased in patients who had been diagnosed with T2DM more than 15 years previously. Studies have shown that with the development of diabetes, image memory and graphic cognitive ability decline [12]. The results of the present study also confirm these phenomena. For example, the degree of carotid stenosis was positively correlated with the severity 
TABLE 2: Binary logistic regression analysis of associations between baseline characteristics and MoCA score in T2DM patients of advanced age.

\begin{tabular}{|c|c|c|c|c|c|c|}
\hline & $\begin{array}{c}\text { Regression } \\
\text { coefficient }(\beta)\end{array}$ & Standard error (SE) & Wald & $P$ & Odds ratio (OR) & $\begin{array}{c}95 \% \text { confidence } \\
\text { interval }(\mathrm{CI}) \\
\end{array}$ \\
\hline \multirow{2}{*}{ Age duration of diabetes (y) } & 1.177 & 1.032 & 2.123 & $0.002^{*}$ & 1.245 & $1.132-3.236$ \\
\hline & 1.321 & 2.143 & 2.876 & $0.025^{*}$ & 1.432 & $1.232-5.441$ \\
\hline BMI $\left(\mathrm{kg} / \mathrm{m}^{2}\right)$ & 1.564 & 1.342 & 5.42 & $0.042^{*}$ & 1.134 & $1.152-3.221$ \\
\hline SBP (mmHg) & 0.464 & 1.302 & 2.434 & 0.221 & 1.589 & $0.878-2.875$ \\
\hline DBP (mmHg) & 0.267 & 2.221 & 0.014 & 0.412 & 1.025 & $0.653-2.624$ \\
\hline Education level (y) & 6.267 & 1.221 & 12.014 & $0.004^{*}$ & 2.025 & $0.953-3.624$ \\
\hline Cardiovascular event & 0.836 & 0.487 & 3.172 & $0.033^{*}$ & 1.122 & $0.162-4.095$ \\
\hline Smoking (\%) & 0.369 & 0.115 & 5.647 & $0.039^{*}$ & 1.463 & $1.161-5.835$ \\
\hline $\mathrm{FBG}(\mathrm{mmol} / \mathrm{L})$ & 1.421 & 0.313 & 2.721 & $0.021^{*}$ & 1.224 & $0.851-2.845$ \\
\hline $\mathrm{HbA1c}(\mathrm{mmol} / \mathrm{L})$ & 2.932 & 0.209 & 0.296 & $0.014^{*}$ & 1.325 & $1.143-4.774$ \\
\hline $\mathrm{SCr}(\mu \mathrm{mol} / \mathrm{L})$ & 0.232 & 5.578 & 1.683 & 0.732 & 4.174 & $1.435-7.456$ \\
\hline BUN (mmol/L) & 0.332 & 6.578 & 2.683 & 0.932 & 1.274 & $0.735-5.456$ \\
\hline $\mathrm{TC}(\mathrm{mmol} / \mathrm{L})$ & 2.345 & 0.421 & 2.532 & $0.027^{*}$ & 5.453 & $0.257-3.052$ \\
\hline $\mathrm{TG}(\mathrm{mmol} / \mathrm{L})$ & 1.622 & 0.426 & 4,643 & $0.035^{*}$ & 2.445 & $0.561-1.625$ \\
\hline $\mathrm{LDL}-\mathrm{C}(\mathrm{mmol} / \mathrm{L})$ & 0.433 & 1.234 & 3.567 & 0.061 & 1.164 & $0.868-2.567$ \\
\hline HDL-C (mmol/L) & 0.632 & 1.578 & 3.683 & 0.532 & 2.574 & $0.435-1.456$ \\
\hline HOMA-IR & 0.142 & 1.765 & 4.643 & 0.417 & 4.245 & $0.153-2.345$ \\
\hline 24 h UP (g/24h) & 3.213 & 4.789 & 5.890 & $0.027^{*}$ & 2.865 & $0.346-7.546$ \\
\hline
\end{tabular}

TABLE 3: Analysis of risk factors for cognitive impairment in T2DM patients of advanced age.

\begin{tabular}{|c|c|c|c|c|c|c|}
\hline & Regression coefficient $(\beta)$ & Standard error (SE) & Wald & $P$ & Odds ratio (OR) & $95 \%$ confidence interval $(\mathrm{CI})$ \\
\hline Age (y) & 5.177 & 0.032 & 2.123 & $0.002^{*}$ & 4.253 & $1.132-3.236$ \\
\hline Duration of diabetes $(y)$ & 3.321 & 0.143 & 2.876 & $0.025^{*}$ & 1.424 & $1.232-5.441$ \\
\hline Smoking (\%) & 2.369 & 1.115 & 10.647 & 0.059 & 3.245 & $1.161-1.835$ \\
\hline BMI $\left(\mathrm{kg} / \mathrm{m}^{2}\right)$ & 1.564 & 0.342 & 5.421 & 0.052 & 0.934 & $1.152-1.221$ \\
\hline Cardiovascular event & -0.836 & 0.487 & 3.172 & 0.073 & 43.567 & 0.162-1.095 \\
\hline Education level (y) & 5.267 & 1.221 & 7.014 & $0.012^{*}$ & 5.356 & $0.453-2.637$ \\
\hline FBG $(\mathrm{mmol} / \mathrm{L})$ & 4.421 & 0.313 & 2.721 & $0.021^{*}$ & 6.435 & $0.851-2.845$ \\
\hline $\mathrm{HbAlc}(\mathrm{mmol} / \mathrm{L})$ & 6.032 & 0.209 & 0.296 & $0.014^{*}$ & 1.956 & $1.143-4.774$ \\
\hline $\mathrm{TC}(\mathrm{mmol} / \mathrm{L})$ & 2.345 & 0.421 & 2.532 & $0.027^{*}$ & 1.345 & $0.257-3.052$ \\
\hline $\mathrm{TG}(\mathrm{mmol} / \mathrm{L})$ & 1.622 & 0.426 & 4,643 & $0.035^{*}$ & 1.643 & $0.561-1.625$ \\
\hline $24 \mathrm{~h}$ UP $(\mathrm{g} / 24 \mathrm{~h})$ & 2.134 & 1.567 & 1.754 & $0.041^{*}$ & 3.175 & $0.386-6.424$ \\
\hline HOMA-IR & 0.142 & 1.765 & 4.643 & 0.417 & 4.245 & $0.153-2.345$ \\
\hline
\end{tabular}

${ }^{*} P<0.05$.

TABLE 4: Correlation between fundus retinopathy and cognitive impairment in T2DM patients of advanced age.

\begin{tabular}{lccccc}
\hline Retinopathy & NCF group $(n=40)$ & MCI group $(n=37)$ & MoCI group $(n=31)$ & SCI group $(n=12)$ & $\chi^{2}$ \\
\hline Mild degree (phase I-II) & 39 & 35 & 28 & 7 & 16.194 \\
Severe degree (phase III-IV) & 1 & 2 & 3 & $5.002^{*}$ \\
\hline
\end{tabular}


TABLE 5: Correlation between macrovascular disease and cognitive impairment in T2DM patients of advanced age.

\begin{tabular}{|c|c|c|c|c|c|c|}
\hline Carotid ultrasound & NCF group $(n=40)$ & MCI group $(n=37)$ & MoCI group $(n=31)$ & SCI group $(n=12)$ & $\chi^{2}$ & $P$ value \\
\hline Mild stenosis & 36 & 33 & 22 & 6 & 14.437 & $0.003^{*}$ \\
\hline Severe stenosis & 4 & 4 & 9 & 6 & & \\
\hline
\end{tabular}

${ }^{*} P<0.05$.

TABLE 6: Correlations between amino acid levels $(\mu \mathrm{mol} / \mathrm{L})$ and cognitive impairment in T2DM patients of advanced age.

\begin{tabular}{|c|c|c|c|c|c|c|}
\hline Amino Acid & NCF group $(n=40)$ & MCI group $(n=37)$ & MoCI group $(n=31)$ & SCI group $(n=12)$ & $F$ & $P$ value \\
\hline Ala & $135.84(111.22,156.64)$ & $134.51(125.86,153,81)$ & $133.43(105.13,149.17)$ & $126.26(100.07,175.67)$ & 1.345 & 0.862 \\
\hline $\operatorname{Arg}$ & $4.19(2.23,7.30)$ & $3.04(2.23,4.13)$ & $2.92(2.05,3.84)$ & $1.10(0.85,3.29)$ & 2.342 & 0.013 \\
\hline Asn & $78.30 \pm 15.78$ & $75.76 \pm 19.57$ & $75.60 \pm 20.34$ & $73.00 \pm 20.13$ & 0.284 & 0.837 \\
\hline Asp & $27.33(18.52,36.89)$ & $18.74(14.17,22.60)$ & $16.40(12.80,18.21)$ & $14.26(13.37,17.08)$ & 2.920 & 0.097 \\
\hline Cit & $20.11(17.94,23.32)$ & $22.17(18.26,25.14)$ & $20.59(17.70,22.58)$ & $16.96(14.89,19.18)$ & 1.585 & 0.167 \\
\hline Orn & $12.39(10.15,15,39)$ & $9.30(8.67,11.06)$ & $9.05(7.53,10.35)$ & $10.11(7.25,9.48)$ & 2.447 & 0.061 \\
\hline Gln & $2.33(7.35,14.09)$ & $4.93(5.75,6.54)$ & $6.01(4.59,5.37)$ & $9.02(2.04,4.11)$ & 6.678 & $0.001^{*}$ \\
\hline Lys & $165.79(130.53,259.59)$ & $106.48(101.65,115.36)$ & $86.26(80.31,93.99)$ & $40.89(35.79,72.03)$ & 3.275 & 0.001 \\
\hline Met & $18.87(16.54,24.10)$ & $16.52(14.85,19.37)$ & $16.21(13.84,18.16)$ & $17.52(14.86,18.94)$ & 2.179 & 0.061 \\
\hline His & $50.67(42.06,190.74)$ & $34.99(32.94,38.69)$ & $43.58(29.07,37.23)$ & $33.66(26.58,33.79)$ & 3.111 & 0.072 \\
\hline Leu & $160.23(138.89,189.05)$ & $134.87(109.26,152.48)$ & $130.26(115.28,146.71)$ & $143.43(111.91,152.58)$ & 1.424 & 0.067 \\
\hline Gly & $172.83(160.11,196.85)$ & $166.34(147.75,178.87)$ & $162.65(153.88,176.33)$ & $171.11(146.03,184.29)$ & 2.532 & 0.087 \\
\hline Glu & $213.57(117.93,140.35)$ & $184.93(101.56,123.83)$ & $132.12(92.05,128.00)$ & $108.34(108.98,250.08)$ & 4.256 & $0.002^{*}$ \\
\hline $\operatorname{Trp}$ & $45.96(41.89,54.74)$ & $42.86(35.50,51.09)$ & $40.58(35.37,47.35)$ & $55.25(40.61,67.82)$ & 2.245 & 0.083 \\
\hline Pro & $608.33(506.93,739.37)$ & $532.34(481.76,688.28)$ & $521.25(421.82,586.46)$ & $529.97(323.26,457.34)$ & 5.920 & $0.044^{*}$ \\
\hline Phe & $36.79(32.60,43.96)$ & $34.17(28.30,36.24)$ & $32.01(28.96,36.99)$ & $31.69(27.87,33.40)$ & 6.645 & $0.003^{*}$ \\
\hline Pip & $400.36 \pm 98.18$ & $368.17 \pm 58.63$ & $377.95 \pm 59.50$ & $364.14 \pm 51.14$ & 4.384 & 0.076 \\
\hline Tyr & $52.02 \pm 10.64$ & $49.02 \pm 9.50$ & $46.29 \pm 12.18$ & $41.32 \pm 8.44$ & 6.420 & $0.020^{*}$ \\
\hline Val & $154.22 \pm 23.46$ & $144.72 \pm 30.18$ & $143.20 \pm 28.37$ & $147.70 \pm 15.14$ & 1.142 & 0.335 \\
\hline Thr & $52.02 \pm 10.64$ & $45.02 \pm 9.50$ & $46.29 \pm 12.10$ & $46.32 \pm 8.44$ & 2.920 & 0.057 \\
\hline Ser & $40.93 \pm 6.67$ & $42.45 \pm 6.55$ & $38.63 \pm 7.25$ & $44.20 \pm 7.92$ & 2.447 & 0.067 \\
\hline Hcy & $11.54 \pm 1.35$ & $10.34 \pm 1.23$ & $9.75 \pm 1.07$ & $6.45 \pm 1.33$ & 5.678 & $0.045^{*}$ \\
\hline Cys & $2.56 \pm 1.03$ & $2.11 \pm 0.98$ & $2.64 \pm 0.94$ & $2.14 \pm 1.09$ & 2.179 & 0.094 \\
\hline Val/Phe & $4.19(3.70,4.55)$ & $4.40(3.78,4.95)$ & $4.28(3.91,4.76)$ & $4.79(4.50,5.15)$ & 3.253 & 0.157 \\
\hline Tyr/Cit & $1.33(1.08,1.68)$ & $1.12(0.95,1.34)$ & $1.14(0.97,1.42)$ & $1.47(1.26,1.79)$ & 2.173 & 0.055 \\
\hline Phe/Tyr & $0.75(0.65,0.86)$ & $0.67(0.63,0.76)$ & $0.70(0.62,0.81)$ & $0.74(0.68,0.84)$ & 2.179 & 0.258 \\
\hline Orn/Cit & $0.57(0.46,0.79)$ & $0.44(0.41,0.56)$ & $0.45(0.40,0.52)$ & $0.50(0.42,0.52)$ & 3.119 & 0.051 \\
\hline Met/Phr & $0.52(0.46,0.59)$ & $0.50(0.45,0.56)$ & $0.48(0.45,0.55)$ & $0.57(0.45,0.57)$ & 3.420 & 0.118 \\
\hline Met/Leu & $0.13(0.12,0.14)$ & $0.13(0.11,0.16)$ & $0.12(0.10,0.13)$ & $0.15(0.11,0.16)$ & 1.132 & 0.303 \\
\hline Gly/Ala & $1.10(0.91,1.35)$ & $1.05(0.88,1.25)$ & $1.13(0.92,1.24)$ & $0.96(0.88,1.23)$ & 2.920 & 0.562 \\
\hline Cit/Arg & $5.30(2.40,9.06)$ & $7.14(5.13,10.24)$ & $7.09(5.13,10.33)$ & $19.13(5.52,21.58)$ & 1.585 & 0.054 \\
\hline Arg/Orn & $0.37(0.24,0.44)$ & $0.28(0.22,0.40)$ & $0.32(0.24,0.42)$ & $0.13(0.11,0.35)$ & 2.447 & 0.085 \\
\hline Val/Phe & $4.19(3.70,4.55)$ & $4.40(3.78,4.95)$ & $4.28(3.91,4.76)$ & $4.79(4.50,5.15)$ & 3.253 & 0.157 \\
\hline Tyr/Cit & $1.33(1.08,1.68)$ & $1.12(0.95,1.34)$ & $1.14(0.97,1.42)$ & $1.47(1.26,1.79)$ & 2.173 & 0.055 \\
\hline
\end{tabular}

${ }^{*} P<0.05$.

of cognitive dysfunction in our patients. Diabetes accelerates vascular aging, reduces cerebral blood flow, causes focal ischemic infarction, leads to diffusion of white matter and basal ganglia, and causes neuronal damage and apoptosis, leading to impaired executive cognitive function $[5,13]$. On the other hand, diabetes can affect microvascular endothelial cell function, leading to the impairment of the blood-brain barrier and neuroinflammatory reactions. Studies have 

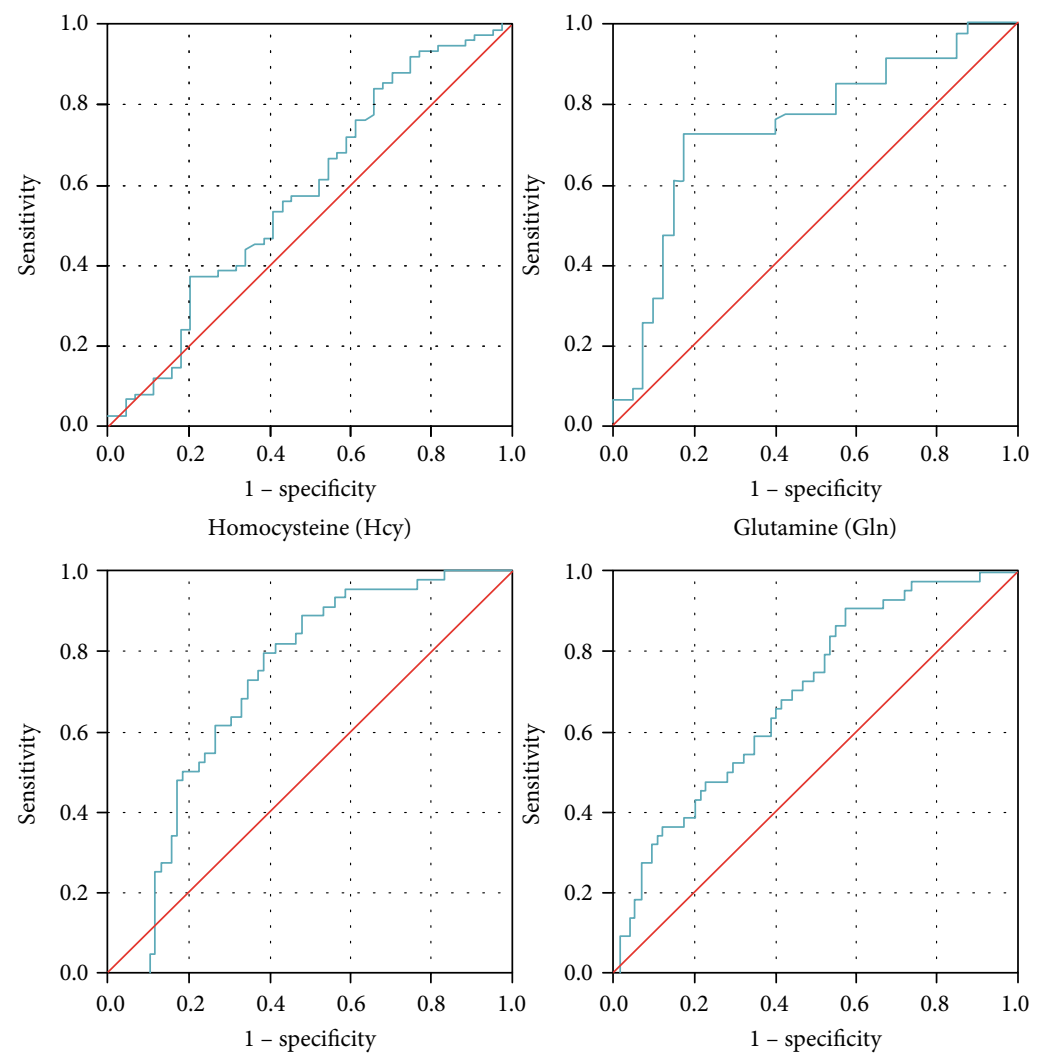

Glutamate (Glu)

Phenylalanine (Phe)
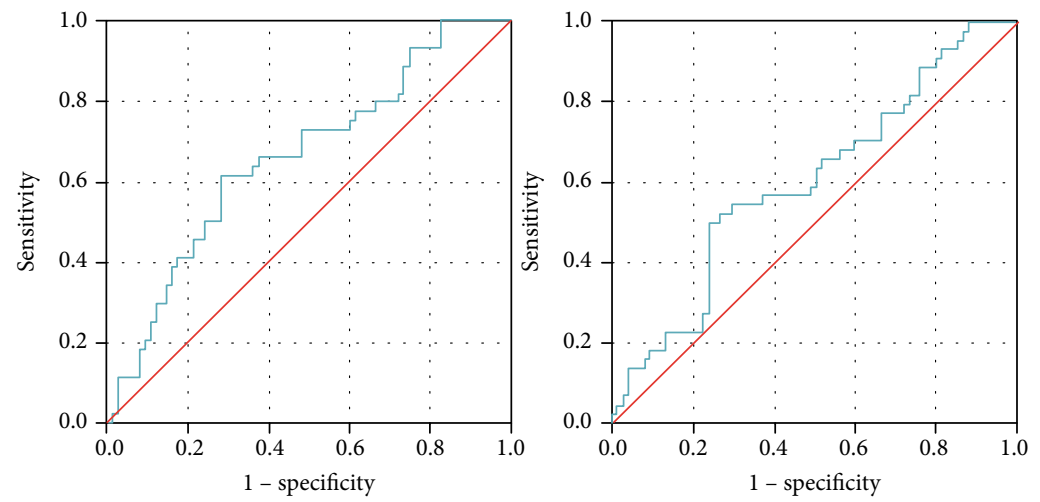

Proline (Pro)

Tyrosine (Tyr)

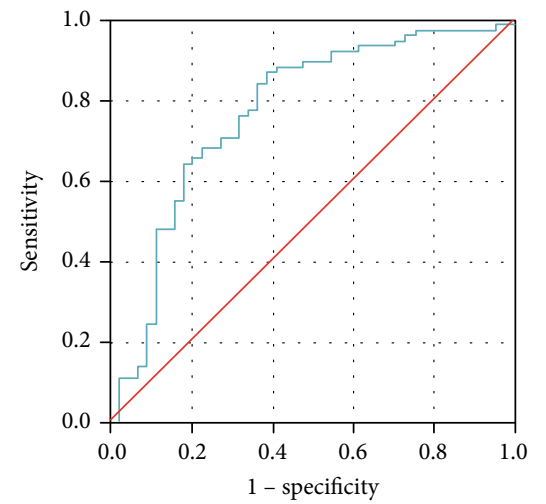

Glutamic acid combined with glutamine (Glu+Gln)

FIGURE 1: ROC curve analysis of the association of amino acid levels with cognitive impairment in T2DM patients of advanced age. 
TABLE 7: ROC curve analysis of the ability of the levels of six amino acids to predict cognitive impairment in T2DM patients of advanced age.

\begin{tabular}{lccccc}
\hline Amino acid & AUC & $P$ value & Cut-point & Sensitivity & Specificity \\
\hline Hcy & 0.581 & 0.142 & 9.500 & 0.840 & 0.341 \\
Gln & 0.739 & $0.001^{*}$ & 5.902 & 0.722 & 0.825 \\
Glu & 0.721 & $0.002^{*}$ & 116.431 & 0.795 & 0.613 \\
Phe & 0.697 & $0.003^{*}$ & 31.746 & 0.909 & 0.427 \\
Pro & 0.661 & $0.004^{*}$ & 598.183 & 0.614 & 0.719 \\
Tyr & 0.602 & 0.064 & 52.174 & 0.501 & 0.747 \\
Gln+Glu & 0.769 & $0.001^{*}$ & 0.610 & 0.867 & 0.614 \\
\hline
\end{tabular}

${ }^{*} P<0.05$.

shown that neurovascular units are related to the dynamics of blood flow in the brain. Changes in microvascular structure, decreased numbers of capillaries, and increased arteriovenous shorts in T2DM patients may affect the transport of nutrients to nerve tissue, and brain tissues are easily damaged by oxygen deficiency when perfusion pressure or blood flow is decreased [13]. Consistently, our data revealed a positive correlation between the staging of fundus retinopathy and the severity of cognitive dysfunction.

Our findings agree with those of other studies showing that hyperglycemia, as accessed by HbAlc concentration, is correlated with cognitive dysfunction in T2DM patients. Long-term hyperglycemia may lead to the thickening of the cerebral vascular muscle basement membrane, reduce cerebral blood circulation, and directly damage neurons. Studies have confirmed that reduced cerebral blood flow obstructs the brain's ability to understand, process, integrate information, etc., ultimately leading to impaired learning and memory ability [14]. One proposed mechanism involves fibronectin (Fn), which is a macromolecular glycoprotein found in plasma and the extracellular matrix that is damaged during periods of hyperglycemia. This damage causes the capillaries within the brain to proliferate, increasing the permeability of the blood-cerebrospinal fluid barrier. More inflammatory cell molecules and antibodies are then allowed to attack brain cells through the blood-cerebrospinal fluid barrier, leading to a decline in cognitive function [15]. In addition, during hyperglycemia, tau protein and amyloid $\beta$ are nonenzymatically glycosylated to form glycosylation end products (AGEs), which are known to participate in the pathological manifestations of Alzheimer's disease.

The present study also identified high levels of TC and TG as risk factors for the development of cognitive impairment in older T2DM patients. T2DM is commonly complicated by lipid metabolism disorder, and abnormal lipid metabolism is also closely related to neurological diseases such as Alzheimer's disease, epilepsy, and Parkinson's disease. Elevated serum TC and TG levels can cause damage to brain vascular endothelial cells and may interfere with the metabolism of amyloid precursor proteins, thereby, accelerating the production and accumulation of $\mathrm{A} \beta$ and leading to cognitive dysfunction [16]. Farr et al. [16] pro- posed that increased TC may affect amino acid receptors, impairing hippocampal synaptic transmission and affecting memory formation. Frias et al. [17] found that increased TG was related to impaired speech knowledge. These studies found that higher TC levels correlated with more severe cognitive impairment.

Amino acid metabolism also directly affects the activity of the nervous system. For example, it was reported that the glutamate level is associated with the level of cognition. Abnormal glutamate metabolism and glutamate receptor function are associated with a variety of neurological diseases including Alzheimer's disease/amnestic mild cognitive impairment (aMCI) [18]. In addition, glutamate production will increase compensatorily to protect the cognitive function in patients with cognitive impairment. The results of our metabolomics analysis showed that Glu, Phe, Tyr, Pro, and Hcy levels increased with the development of cognitive impairment, while the Gln level decreased. Proline is a metabolite of glutamate. The upregulation of proline may be related to abnormal protein aggregation in the brain and abnormal glutamate-proline metabolic signaling [19]. Tyrosine, phenylalanine, and phenethylamine are sulfurcontaining amino acids or related products involved in the metabolism of various neurotransmitters. When cognitive impairment begins to occur, there may also be compensatory mechanisms that promote the upregulation of these neurotransmitter precursors to supplement the lack of neurotransmitters in the brain [20]. Many reports have indicated that hyperhomocysteinemia is common in patients with T2DM. This may be due to the lack of insulin in T2DM patients, which affects the catabolism of homocysteine. Moreover, hyperhomocysteinemia may be a risk factor for cognitive decline [20].

Additionally, it is notable that the level of Gln was negatively correlated with cognitive dysfunction in our statistical analysis, and we believe this may be related to the course of diabetes. Studies have shown that glutamate metabolism is closely related to insulin resistance. Tulipani et al. [21] found a significant increase in the glutamate level in 64 patients with morbid obesity and early-stage diabetes, which was proportional to the insulin resistance index in these patients. Takashina et al. [22] also showed that the glutamate level was increased while the glutamine level was decreased in 83 obese patients. Concomitantly, HOMA-IR and HOMA- $\beta$ were directly proportional to glutamate levels and inversely proportional to glutamine levels. In animal studies, Perdigon et al. [23] observed a decline in cognitive function in high-fat diet-fed rats and a concomitant decline in glutamate levels.

Diabetes is closely associated with the occurrence of cognitive dysfunction or dementia. However, there is no unified diagnostic standard for diabetic cognitive dysfunction currently. Diabetes-related cognitive disorders are often overlooked in the diagnosis and treatment process, resulting in many diabetic patients with cognitive impairment suffering from diminishing memory for a long time [24-26]. Our study analyzed the data from 120 diabetic patients and confirmed some clinical features and risk factors related to cognitive decline. In addition, according to existing reports, there are many risk factors for cognitive decline in type 2 
diabetes patients, including diabetes-specific factors, demographic factors, genetic factors, and lifestyle factors. Nevertheless, there has been no clinical research discussing the effect of amino acid metabolism on the process of diabetes cognitive dysfunction. Amino acids, as the basic units of protein, participate in the synthesis and metabolism of various active substances in the body and play important roles in learning, memory, and nerve conduction. This study examined cognition-related amino acid metabolites in 120 diabetic patients, and such research is very important for the discovery of valuable biomarkers for early diagnosis of diabetesrelated cognitive impairment.

The present study has a number of limitations that should be considered. First, this was a cross-sectional study with a small sample size, which may limit the reliability of the results. Next, we did not exclude individuals who had recently received medicines that could affect cognitive function. Furthermore, we did not collect data related to daily exercise, which has been shown to strongly affect the level of cognitive impairment. Additionally, we did not include food recall in our questionnaire because of inconvenient operation, and any changes in food intake can have a considerable influence on the cognitive index. Thus, this is a limitation in our research design as well. Finally, we did not investigate the influence of hypoglycemia on the cognitive function of T2DM patients. Current research results are controversial, and due to the recall bias introduced by patients' ability to recall of hypoglycemic events, we did not collect information regarding hypoglycemia.

\section{Conclusion}

In summary, age, educational level, duration of diabetes, and levels of FBG, HbAlc, TC, TG, and 24-hour urine protein were identified as independent risk factors for cognitive impairment in T2DM patients of advanced age (50-70 years). Macrovascular and microvascular diseases also were strongly associated with cognitive decline in these patients. The levels of glutamate, glutamine, proline, and phenylalanine may be good predictive biomarkers of cognitive impairment in T2DM patients of advanced age, and together, glutamate and glutamine levels may represent the most effective biomarker panel among the identified amino acids. This finding could have an important clinical impact on the search for predictive serological markers for early diagnosis of cognitive impairment in T2DM patients. However, further investigation of the value of these predictors in practical application is warranted.

\section{Data Availability}

The data used to support the findings of this study are available from the corresponding author upon request.

\section{Ethical Approval}

The study was reviewed and approved by the ethics committee of the 1st Hospital of Jilin University.

\section{Consent}

The patients provided written informed consent for participation in this study.

\section{Conflicts of Interest}

The authors declare no conflict of interest.

\section{Acknowledgments}

Funding for this work was obtained from the Open Topic Foundation of Science and Technology Department of Jilin Province (No. 20170623092TC-03 and No. 20180623083T C-03).

\section{Supplementary Materials}

Table A1: reference ranges for indicators. Table A2: correlation between level of acylcarnitine $(\mu \mathrm{mol} / \mathrm{L})$ and cognitive impairment in T2DM patients of advanced age. (Supplementary materials)

\section{References}

[1] M. Stumvoll, B. J. Goldstein, and T. W. van Haeften, "Type 2 diabetes: principles of pathogenesis and therapy," Lancet, vol. 365, no. 9467, pp. 1333-1346, 2005.

[2] P. Zimmet, K. G. Alberti, and J. Shaw, "Global and societal implications of the diabetes epidemic," Nature, vol. 414, no. 6865 , pp. 782-787, 2001.

[3] G. J. Biessels, I. J. Deary, and C. M. Ryan, "Cognition and diabetes: a lifespan perspective,” Lancet Neurology, vol. 7, no. 2, pp. 184-190, 2008.

[4] J. Tang, Y. Pei, and G. Zhou, "When aging-onset diabetes is coming across with Alzheimer disease: comparable pathogenesis and therapy," Experimental Gerontology, vol. 48, no. 8, pp. 744-750, 2013.

[5] R. Stewart and D. Liolitsa, "Type 2 diabetes mellitus, cognitive impairment and dementia," Diabetic Medicine, vol. 16, no. 2, pp. 93-112, 1999.

[6] T. Cukierman, H. C. Gerstein, and J. D. Williamson, "Cognitive decline and dementia in diabetes-systematic overview of prospective observational studies," Diabetologia, vol. 48, no. 12, pp. 2460-2469, 2005.

[7] R. H. X. Wong, A. Scholey, and P. R. C. Howe, "Assessing premorbid cognitive ability in adults with type 2 diabetes mellitus-a review with implications for future intervention studies," Current Diabetes Reports, vol. 14, no. 11, p. 547, 2014.

[8] H. J. Lee, H. I. Seo, H. Y. Cha, Y. J. Yang, S. H. Kwon, and S. J. Yang, "Diabetes and Alzheimer's disease: mechanisms and nutritional aspects," Clin Nutr Res., vol. 7, no. 4, pp. 229240, 2018

[9] C. Qiu, B. Winblad, and L. Fratiglioni, "The age-dependent relation of blood pressure to cognitive function and dementia," Lancet Neurology, vol. 4, no. 8, pp. 487-499, 2005.

[10] O. Hansson, H. Zetterberg, P. Buchhave, E. Londos, K. Blennow, and L. Minthon, "Association between CSF biomarkers and incipient Alzheimer's disease in patients with mild cognitive impairment: a follow-up study," Lancet Neurology, vol. 5, no. 3, pp. 228-234, 2006. 
[11] G. Logroscino, J. H. Kang, and F. Grodstein, "Prospective study of type 2 diabetes and cognitive decline in women aged 70-81 years," BMJ, vol. 328, no. 7439, p. 548, 2004.

[12] R. Cosway, M. W. Strachan, A. Dougall, B. M. Frier, and I. J. Deary, "Cognitive function and information processing in type 2 diabetes," Diabetic Medicine, vol. 18, no. 10, pp. 803-810, 2001.

[13] N. Yuen, S. E. Anderson, N. Glaser, D. J. Tancredi, and M. E. O'Donnell, "Cerebral blood flow and cerebral edema in rats with diabetic ketoacidosis," Diabetes, vol. 57, no. 10, pp. 2588-2594, 2008.

[14] T. A. Enache and A. M. Oliveira-Brett, "Alzheimer's disease amyloid beta peptides in vitro electrochemical oxidation," Bioelectrochemistry, vol. 114, pp. 13-23, 2017.

[15] A. Ergul, M. M. Elgebaly, M. L. Middlemore et al., "Increased hemorrhagic transformation and altered infarct size and localization after experimental stroke in a rat model type 2 diabetes," BMC Neurology, vol. 7, no. 1, 2007.

[16] S. A. Farr, K. A. Yamada, D. A. Butterfield et al., "Obesity and hypertriglyceridemia produce cognitive impairment," Endocrinology, vol. 149, no. 5, pp. 2628-2636, 2008.

[17] C. M. de Frias, D. Bunce, A. Wahlin et al., "Cholesterol and triglycerides moderate the effect of apolipoprotein E on memory functioning in older adults," The Journals of Gerontology. Series B, Psychological Sciences and Social Sciences, vol. 62, no. 2, pp. P112-P118, 2007.

[18] M. Emre, P. J. Ford, B. Bilgic, and E. Y. Uc, "Cognitive impairment and dementia in Parkinson's disease: practical issues and management," Movement Disorders, vol. 29, no. 5, pp. 663672, 2014.

[19] G. Trifirò, M. M. Mokhles, J. P. Dieleman et al., "Risk of cardiac valve regurgitation with dopamine agonist use in Parkinson's disease and hyperprolactinaemia: a multi-country, nested casecontrol study," Drug Safety, vol. 35, no. 2, pp. 159-171, 2012.

[20] R. Obeid, A. McCaddon, and W. Herrmann, "The role of hyperhomocysteinemia and B-vitamin deficiency in neurological and psychiatric diseases," Clinical Chemistry and Laboratory Medicine, vol. 45, no. 12, pp. 1590-1606, 2007.

[21] S. Tulipani, M. Palau-Rodriguez, A. M. Alonso et al., "Biomarkers of morbid obesity and prediabetes by metabolomic profiling of human discordant phenotypes," Clinica Chimica Acta, vol. 463, pp. 53-61, 2016.

[22] C. Takashina, I. Tsujino, T. Watanabe et al., "Associations among the plasma amino acid profile, obesity, and glucose metabolism in Japanese adults with normal glucose tolerance," Nutr Metab (Lond), vol. 13, no. 1, 2016.

[23] M. Rodriguez-Perdigon, M. Solas, M. J. Moreno-Aliaga, and M. J. Ramirez, "Lipoic acid improves neuronal insulin signalling and rescues cognitive function regulating VGlut1 expression in high-fat-fed rats: implications for Alzheimer's disease," Biochimica et Biophysica Acta (BBA) - Molecular Basis of Disease, vol. 1862, no. 4, pp. 511-517, 2016.

[24] Y. D. Reijmer, E. van den Berg, C. Ruis, L. J. Kappelle, and G. J. Biessels, "Cognitive dysfunction in patients with type 2 diabetes," Diabetes/Metabolism Research and Reviews, vol. 26, no. 7, pp. 507-519, 2010.

[25] L. A. Zilliox, K. Chadrasekaran, J. Y. Kwan, and J. W. Russell, "Diabetes and cognitive impairment," Current Diabetes Reports, vol. 16, no. 9, p. 87, 2016.

[26] H. Umegaki, "Type 2 diabetes as a risk factor for cognitive impairment: current insights," Clinical Interventions in Aging, vol. 9, pp. 1011-1019, 2014. 